\title{
Journal of Gynecology and Obstetrics Bulletin
}

\author{
Genomic Analysis of Sarcomagenesis \\ Takuma Hayashi ${ }^{1,7,8^{*}}$, Akiko Horiuchi ${ }^{2}$, Yae Kanai ${ }^{3,9}$, Nobuo Yaegashi ${ }^{4}$, Susumu Tonegawa ${ }^{5}$ and Ikuo Konishi ${ }^{6}$ \\ ${ }^{1}$ Department of Immunology and Infectious Disease, Shinshu University School of Medicine, Nagano Japan \\ ${ }^{2}$ Horiuchi Ladies Clinic, Nagano Japan \\ ${ }^{3}$ Department of Pathology, Keio University, School of Medicine, Tokyo Japan \\ ${ }^{4}$ Department of Obstetrics and Gynecology, Tohoku University Graduate School of Medicine, Nagano Japan \\ ${ }^{5}$ Picower Institute for Learning and Memory and Massachusetts Institute of Technology, MA, USA \\ ${ }^{6}$ Department of Obstetrics and Gynecology, Kyoto University Graduate School of Medicine, Kyoto, Japan \\ 7Promoting Business using Advanced Technology, Japan Science and Technology Agency (JST), Tokyo, Japan \\ ${ }^{8}$ Sigma-Aldrich Collaboration Laboratory, Rehovot, Israel
}

${ }^{9}$ The International Human Epigenome Consortium (IHEC) and CREST, Japan Science and Technology Agency (JST), Saitama, Japan

\begin{abstract}
*Corresponding author: Takuma Hayashi, Department of Immunology and Infectious Disease, Shinshu University Graduate School of Medicine,3-1-1, Asahi, Matsumoto, Nagano 390-8621, Japan; Tel: 81-263-37-2611; E mail: yoyoyo224@hotmail.com
\end{abstract}

Article Type: Short Communication, Submission Date: 28 December 2015, Accepted Date: 13 January 2016, Published Date: 03 February 2016.

Citation: Takuma Hayashi, Akiko Horiuchi, Yae Kanai, Nobuo Yaegashi, Susumu Tonegawa and Ikuo Konishi (2016) Genomic Analysis of Sarcomagenesis. JGynObsBul 1(1): 1-2.

Copyright: (c) 2016 Takuma Hayashi, et al. This is an open-access article distributed under the terms of the Creative Commons Attribution License, which permits unrestricted use, distribution, and reproduction in any medium, provided the original author and source are credited.

\begin{abstract}
Sarcomas are neoplastic malignancies that typically arise in mesenchymal tissues. Like most genetic diseases, this type of cancer is rarely observed in less than 15,000 new cases per year in the United States. The identification of molecular mechanisms generating sarcomas and developing a new tests and therapies are complicated by very heterogeneous Sarcomas arising in many tissue lineages. Thus analyzing a substantial frequency of specific clinical samples requires analysis of extensive total patient populations. Mouse models genomes have been tailored with gene deletions, amplifications, and point mutations reported in human sarcomas to minimize the number of human patients required.Given that $\sim 80 \%$ of mouse mutations result in similar sarcomagenesis and suppressive therapies, confirmation in humans is required. Thus mouse models serve as powerful in vivomodels to establish new biomarkers and develop therapies.
\end{abstract}

Keywords:Leiomyosarcoma; LMP2; Tumor protein 53 (TP53); Retinoblastoma (Rb).

\section{Introduction}

Malignant sarcoma tumors are highly debilitating and significantly associated with morbidity and mortality. Defined sarcomas arise from a plethora of tissues that are additionally stratified by their histopathology or patient's age at diagnosis[1]. Modern rapid genomewide, pathobiological and clinical analysis further define and stratify sarcomas [2]. Cytogenetic analyses have revealed two divergent genetic profiles. The first karyotypic profile includes about a dozen specific translocations that initiate a specific cancer of all categories.In comparison, most sarcomas display a more complex genotype consistent with more complex phenotypes and rapidly advancing oncogenic tissues.

\section{IFN-b-inducible factor $\mathrm{LMP2/b1i}$ related to uterine mesenchymal transformation}

Proteasomal degradation is essential for multiple cellular categories including the modulation of cell cycle, gene expression, and immunological function [3,4,5]. Interferon (IFN)- $b$ induces the expression of large numbers of responsive genes including subunits of the proteasome b-ring, i.e., lowmolecular mass polypeptide (LMP)2/b1i, LMP7/b5i, and LMP10/ multicatalyticendopeptidase complex-like (MECL)-1/b2i [7]. A molecular approach to investigating the relationship between IFN- $\gamma$ and tumor cell growth has been enhanced by genomewide sequencing. Homozygous mice deficient in $1 \mathrm{mp} 2 / \mathrm{b} 1 \mathrm{i}$ show tissueand substrate-dependent abnormalities in proteasome functions [7]. Uterine leiomyosarcoma (Ut-LMS) reportedly occurred in female $L m p 2 / \mathrm{b} 1 i$-deficientmice beginning at 6 months of age, to an incidence of $37 \%$ at 12 months of age [8]. Histological studies on $\operatorname{lmp} 2 /$ b1i-without uterine tumors have characteristic Ut-LMS abnormalities [8].Recent study, experiments with mouse and human uterine tissues revealed a defective human LMP2/b1i expression in Ut-LMS in the IFN-b pathway along with specific effect of JANUS KINASE 1 (JAK1) somatic mutations on the LMP2/b1i transcriptional activation [9]. Furthermore, analysis of a human Ut-LMS cell line clarified the biological significance of LMP2/b1i in malignant myometrium transformation, implicating LMP2/b1i as an anti-tumorigenic candidate $[9,10]$.

\section{Tumor suppressors and oncogenic pathways involved in sarcomagenesis}

The tumor protein 53 (TP53) tumor suppressor pathway is a well characterized signal cascade in tumorigenesis[11]. TP53 is a transcription regulator gene that activates numerous DNA 
Citation: Takuma Hayashi, Akiko Horiuchi, Yae Kanai, Nobuo Yaegashi, Susumu Tonegawa and Ikuo Konishi (2016) Genomic Analysis of Sarcomagenesis. JGynObsBul 1(1): 1-2.

damage-dependent checkpoint responses plus apoptotic genes that ablate many malignant tumors. In addition to TP53 loss of function via inherited mutations, the TP53 pathway is commonly disrupted during sporadic sarcomagenesis [12,13]. However, a substantial proportion of sarcomas retain wild-type TP53 but phenotypically display a loss of TP53 function. These research findings suggest that changes in other components of the TP53 signal cascade; such as amplification of MDM2, a negative regulator of TP53 pathway, may result in TP53 inactivation[14,15]. Furthermore, mice and humans with elevated levels of MDM2 due to a common single nucleotide polymorphism in the MDM2 promoter (MDM2SNP309) are both more susceptible to sarcoma formation [16]. Additionally, deletion or silencing of $p 19^{A r f}$, an inhibitor of the MDM2-TP53 axis, often results in development of sarcomas. Together, these findings indicate that while inactivation of the TP53 pathway is observed in the vast majority of human sarcomas, the mechanisms that disrupt of the pathway vary greatly.

The Retinoblastoma (Rb) [17] pathway is a second major tumor suppressor pathway that is deregulated in many sarcomas. Individuals inheriting a germline $R B 1$ gene mutation typically develop retinal cancer early in life. In addition these children have a significantly higher propensity to develop sarcomas than the general population [18]. While the inheritance of germlineRB1alterations increases the risk of sarcoma, there are also numerous examples of sporadic sarcomas harboring spontaneous mutations and deletions in $R B$, particularly osteosarcomas and rhabdomyosarcomas [19]. Furthermore, $P 16^{I N K 4 A}$, a negative regulator of the CDK-CYCLIN complexes that phosphorylate and activate $R B 1$, is often deleted in sarcomas[20]. Together, these findings illustrate the importance of $\mathrm{Rb}$ pathways in sarcomagenesis.

\section{Conclusion}

The substantial differences in the cellular origins of sarcomas, the lack of availability of tumor specimens in small medical practices, and the heterogeneity within individual tumors has impeded the thorough understanding of sarcoma biology. The availability of numerous genetic knock-outs, knock-ins, and conditional alleles coupled with tissue-specific Cre-recombinase expressing mouse lines, enables determining the impact of individual genes and mutations in mouse sarcomagenesis. Going forward, tumor analysis from multiple murine-derived tumor types can be readily compared and contrasted to identify critical changes in specific sarcomas. Molecular approaches have clearly demonstrated that while there are driver mutations including translocations, sarcomagenesis is a multi-hit disease. These mechanisms in animals can then be tested in the minimal number of human subjects to determine whether the same principles apply. The use of these mouse models mimicking the human disease condition will enhance defining critical therapeutic approaches that impact treating these debilitating diseases.

\section{References}

1. Lasota J, Fanburg-Smith JC. Genetics for the diagnosis and treatment of mesenchymal tumors. SeminMusculoskeletRadiol. 2007; 11(3):215-30.doi: 10.1055/s-2008-1038311.

2. Taylor BS, Barretina J, Maki RG, Antonescu CR, Singer S, Ladanyi M. Advances in sarcoma genomics and new therapeutic targets. Nat Rev Cancer. 2011; 11(8):541-57.doi: 10.1038/nrc3087.
3. Peters JM, Franke WW, Kleinschmidt JA. Distinct $19 \mathrm{~S}$ and $20 \mathrm{~S}$ subcomplexes of the $26 \mathrm{~S}$ proteasome and their distribution in the nucleus and the cytoplasm.J Biol Chem. 1994; 269(10):7709-18.

4. Lodish H, Berk A, Matsudaira P, Kaiser CA, Krieger M, Scott MP, et al. 2004 " 3 ". Mol Cell Biol. 5th ed. New York: WH Freeman and CO. 2004; 5:66-72.

5. Konstantinova IM, Tsimokha AS, Mittenberg AG. Role of proteasomes in cellular regulation. Int Rev Cell Mol Biol. 2008; 267:59-124.doi: 10.1016/S1937-6448(08)00602-3.

6. Wang J, Maldonado MA. The Ubiquitin-Proteasome System and Its Role in Inflammatory and Autoimmune Diseases. Cell Mollmmunol. 2006; 3(4):255-61.

7. Van Kaer L, Ashton-Rickardt PG, Eichelberger M, Gaczynska M, Nagashima K, Rock KL, et al. Altered peptidase and viral-specifi c T cell response in LMP2 mutant mice. Immunity. 1994; 1(7):533-41.

8. Hayashi T, Faustman DL. Development of spontaneous uterine tumors in low molecular mass polypeptide-2 knockout mice. Cancer Res. 2002; 62(1):24-7.

9. Hayashi T, Horiuchi A, Sano K, Hiraoka N, Kasai M, Ichimura T, et al. Potential role of LMP2 as tumor-suppressor defines new targets for uterine leiomyosarcoma therapy. Sci Rep. 2011; 1:180.doi: 10.1038/srep00180.

10. Hayashi T, Horiuchi A, Sano K, Hiraoka N, Kasai M, Ichimura T, et al. Potential role of LMP2 as an anti-oncogenic factor in human uterine leiomyosarcoma: morphological significance of calponin h1. FEBS Lett. 2012; 586(13):1824-1831.doi: 10.1016/j.febslet.2012.05.029.

11. Vogelstein B, Lane D, Levine AJ. Surfing the p53 network. Nature. 2000; 408(6810):307-10.

12. Raycroft L, Wu HY, Lozano G. Transcriptional activation by wild-type but not transforming mutants of the p53 anti-oncogene. Science. 1990; 249(4972):1049-51.

13. Wang LL. Biology of osteogenic sarcoma. Cancer J. 2005; 11(4):294305.

14. Oliner JD, Kinzler KW, Meltzer PS, George DL, Vogelstein B. Amplification of a gene encoding a $\mathrm{p} 53$-associated protein in human sarcomas. Nature. 1992; 358(6381):80-3.

15. Oliner JD, Pietenpol JA, Thiagalingam S, Gyuris J, Kinzler KW, Vogelstein B. Oncoprotein MDM2 conceals the activation domain of tumour suppressor p53. Nature. 1993; 362(6423):857-60.

16. Ito M, Barys L, O'Reilly T, Young S, Gorbatcheva B, Monahan J, et al. Comprehensive Mapping of p53 Pathway Alterations Reveals an Apparent Role for Both SNP309 and MDM2 Amplification in Sarcomagenesis.Clin Cancer Res. 2011; 17(3):416-26.doi: 10.1158/1078-0432.CCR-10-2050.

17. Deshpande A, Hinds PW. The retinoblastoma protein in osteoblast differentiation and osteosarcoma. CurrMol Med. 2006; 6(7):80917.

18. Lohmann DR, Gallie BL. Retinoblastoma. GeneTests. Available from: http://www.ncbi.nlm.nih.gov/books/NBK1452/

19. Toguchida J, Ishizaki K, Sasaki MS, Nakamura Y, Ikenaga M, Kato M, et al. Preferential mutation of paternally derived RB gene as the initial event in sporadic osteosarcoma. Nature. 1989; 338(6211):156-8.

20. Oda Y, Yamamoto H, Takahira T, Kobayashi C, Kawaguchi K, Tateishi N, et al. Frequent alteration of p16(INK4a)/p14(ARF) and p53 pathways in the round cell component of myxoid/round cell liposarcoma: p53 gene alterations and reduced p14(ARF) expression both correlate with poor prognosis. J Pathol. 2005; 207(4):410-21.

JGynObsBul 1(1). 